\title{
Space-Time Block Codes from Cyclic Design
}

\author{
Liang Xian and Huaping Liu, Member, IEEE
}

\begin{abstract}
It has been proved that full-rate complex orthogonal space-time block codes do not exist for systems with more than two transmit antennas. In this letter, we present a space-time block coding scheme based on cyclic design. The proposed codes provide full rate and full diversity for quaternary phase shift keying (QPSK) symbols in systems with three or four transmit antennas.
\end{abstract}

Index Terms-Space-time block codes, spatial diversity, wireless communications.

\section{INTRODUCTION}

$\mathbf{L}$ INEAR orthogonal space-time block codes (STBC) are attractive because of their inter-symbol interference (ISI) free structure and the feasibility of realizing maximum likelihood (ML) decoding using linear operations. However, in [1] Tarokh et. al. proved that full-rate complex orthogonal codes do not exist for systems with more than two transmit antennas. So the Alamouti scheme [2] is the only complex orthogonal code with full transmission rate. In order to achieve full transmission rate in systems with more than two transmit antennas, one must give up orthogonality. Therefore in [3] Jafarkhani proposed a quasi-orthogonal code with full rate but partial diversity for systems with four transmit antennas. In [4] Sharma et. al. proposed a constellation-rotation scheme to improve the performance of quasi-orthogonal code, which was subsequently extended for systems with an arbitrary number of transmit antennas in [5]. Later, He et. al. [6] proposed a nonlinear orthogonal code with full rate and full diversity for QPSK systems with four transmit antennas. However, the encoding and decoding complexities of this code are higher than those of quasi-orthogonal codes.

In this letter, we present a new space-time block coding scheme based on cyclic design. We will first analyze the determinants of real square orthogonal code matrices and propose a new real code from cyclic design. Then, we minimize ISI to design complex cyclic codes that achieve full diversity. We also study the performance of the proposed scheme and compare it with existing schemes.

\section{SySTEM Model}

Consider a system with $N$ transmit antennas and $M$ receive antennas. The transmission matrix is defined as an $L \times N$ matrix $\mathcal{G}(\boldsymbol{x})$, where $L$ is the frame length. The input symbol vector is expressed as $\boldsymbol{x}=\left[x_{1}, \cdots, x_{P}\right]^{T}$, where $(\cdot)^{T}$ denotes transpose and $P$ is number of symbols to be transmitted in

Manuscript received July 20, 2004. The associate editor coordinating the review of this letter and approving it for publication was Prof. G. Taricco.

The authors are with the School of Electrical Engineering and Computer Science, Oregon State University, Corvallis, OR, USA (e-mail: hliu@eecs.orst.edu).

Digital Object Identifier 10.1109/LCOMM.2005.03018. each frame. The codeword corresponding to input symbol vector $\boldsymbol{x}$ is written as $\boldsymbol{c}=c_{1}^{1} \cdots c_{1}^{N} c_{2}^{1} \cdots c_{2}^{N} \cdots c_{L}^{1} \cdots c_{L}^{N}$, where $c_{t}^{n}, n=1,2, \cdots, N, t=1,2, \cdots, L$, is a combination of input constellation symbols. At each time slot $t$, signal $c_{t}^{n}$ is transmitted from antenna $n$. The transmission rate is defined as $R=P / L$.

The channel is assumed to be frequency nonselective Rayleigh, and is modeled as quasi-static, allowing fading coefficients to be constant over a block of data and change independently from one block to another. Let $h_{n, m}, n=$ $1, \cdots, N, m=1, \cdots, M$, be the path gain from transmit antenna $n$ to receive antenna $m$. The path gains are modeled as samples of independent zero-mean complex Gaussian random variables with variance 0.5 per real dimension.

At time $t$, the signal received at antenna $m, r_{t}^{m}$, is given by

$$
r_{t}^{m}=\sum_{n=1}^{N} \sqrt{\frac{E_{s}}{N}} h_{n, m} c_{t}^{n}+\eta_{t}^{m}
$$

where $E_{s}$ is the average energy per symbol and $1 / N$ is the power scaling factor for each transmit antenna so that the total transmission power is normalized. The received noise components $\eta_{t}^{m}, m=1, \cdots, M$, are independent samples of zero-mean complex Gaussian random variables with variance $\mathcal{N}_{0} / 2$ per real dimension. Assuming the availability of perfect channel state information, the receiver computes the following decision metric

$$
\begin{aligned}
d= & \sum_{t=1}^{L} \sum_{m=1}^{M}\left|r_{t}^{m}-\sum_{n=1}^{N} \sqrt{\frac{E_{s}}{N}} h_{n, m} c_{t}^{n}\right|^{2} \\
= & \sum_{m=1}^{M}\left(\boldsymbol{r}_{m}^{H} \boldsymbol{r}_{m}-\sqrt{\frac{E_{s}}{N}} \boldsymbol{h}_{m}^{H} \mathcal{G}^{H}(\boldsymbol{x}) \boldsymbol{r}_{m}-\sqrt{\frac{E_{s}}{N}} \boldsymbol{r}_{m}^{H} \mathcal{G}(\boldsymbol{x}) \boldsymbol{h}_{m}\right. \\
& \left.+\frac{E_{s}}{N} \boldsymbol{h}_{m}^{H} \mathcal{G}^{H}(\boldsymbol{x}) \mathcal{G}(\boldsymbol{x}) \boldsymbol{h}_{m}\right)
\end{aligned}
$$

where $(\cdot)^{H}$ denotes complex conjugate transpose, $\boldsymbol{r}_{m}=$ $\left[r_{1}^{m}, r_{2}^{m}, \cdots, r_{L}^{m}\right]^{T}$, and $\boldsymbol{h}_{m}=\left[h_{1, m}, h_{2, m}, \cdots, h_{N, m}\right]^{T}$. After comparing over all possible codewords, the receiver decides in favor of the codeword that minimizes $d$.

\section{New Codes From CyClic Design}

Cyclic codes are easy to design across time and space domains. Because of a lack of orthogonality, interference will degrade their performance. For this reason, cyclic codes are much less attractive than orthogonal codes from HurwitzRadon family [1] for real symbols. For full-rate complex code design, however, cyclic codes could be very attractive because ISI in these codes may not cause a loss in diversity under some conditions, and full diversity can be achieved without constellation rotation. 
Let us review the rank criterion given in [1], [7]. Let an input symbol vector be $\boldsymbol{y}=\left[y_{1}, y_{2}, \cdots, y_{P}\right]^{T}$, which generates codeword $e=e_{1}^{1} \cdots e_{1}^{N} e_{2}^{1} \cdots e_{2}^{N} \cdots e_{L}^{1} \cdots e_{L}^{N}$. In order to achieve the maximum diversity of order $N M$, the difference matrix between the two code matrices corresponding to distinct input symbol vectors $\boldsymbol{x}$ and $\boldsymbol{y}$

$$
B(\boldsymbol{e}, \boldsymbol{c})=\left(\begin{array}{cccc}
e_{1}^{1}-c_{1}^{1} & e_{1}^{2}-c_{1}^{2} & \cdots & e_{1}^{N}-c_{1}^{N} \\
e_{2}^{1}-c_{2}^{1} & e_{2}^{2}-c_{2}^{2} & \cdots & e_{2}^{N}-c_{2}^{N} \\
\vdots & \vdots & \ddots & \vdots \\
e_{L}^{1}-c_{L}^{1} & e_{L}^{2}-c_{L}^{2} & \cdots & e_{L}^{N}-c_{L}^{N}
\end{array}\right)
$$

must be of full rank. If $B(\boldsymbol{e}, \boldsymbol{c})$ has a minimum rank $r$, then a diversity of order $r M$ is achieved.

\section{A. The determinant criterion}

The determinant criterion for linear space-time codes to achieve full diversity in Rayleigh fading environments: If a square code matrix $\mathcal{G}(\boldsymbol{x})$ is chosen to be such that $\operatorname{det}(\mathcal{G}(\boldsymbol{x})) \neq 0$ for an arbitrary non-zero input vector $\boldsymbol{x}$ (elements of $\boldsymbol{x}$ do not necessarily be constellation symbols), then the code achieves full diversity.

Proof: Because of linearity, $B(\boldsymbol{e}, \boldsymbol{c})=\mathcal{G}(\boldsymbol{y})-\mathcal{G}(\boldsymbol{x})=$ $\mathcal{G}(\boldsymbol{y}-\boldsymbol{x})$. For $\boldsymbol{y} \neq \boldsymbol{x}$, we have $\operatorname{det}(\mathcal{G}(\boldsymbol{y}-\boldsymbol{x})) \neq 0$. Therefore, $B(\boldsymbol{e}, \boldsymbol{c})$ is a full-rank matrix for any pair of distinct codewords $e$ and $c$.

A non-zero determinant is a stronger condition than the fullrank criterion for achieving full diversity. It will be interesting to analyze the determinants of the linear square orthogonal transmission matrices given in [2] and [1]:

$$
\begin{aligned}
& \mathcal{G}_{2}=\left(\begin{array}{cc}
x_{1} & x_{2} \\
-x_{2}^{*} & x_{1}^{*}
\end{array}\right), \quad \mathcal{G}_{4}=\left(\begin{array}{cccc}
x_{1} & x_{2} & x_{3} & x_{4} \\
-x_{2} & x_{1} & -x_{4} & x_{3} \\
-x_{3} & x_{4} & x_{1} & -x_{2} \\
-x_{4} & -x_{3} & x_{2} & x_{1}
\end{array}\right), \\
& \mathcal{G}_{8}=\left(\begin{array}{cccccccc}
x_{1} & x_{2} & x_{3} & x_{4} & x_{5} & x_{6} & x_{7} & x_{8} \\
-x_{2} & x_{1} & x_{4} & -x_{3} & x_{6} & -x_{5} & -x_{8} & x_{7} \\
-x_{3} & -x_{4} & x_{1} & x_{2} & x_{7} & x_{8} & -x_{5} & -x_{6} \\
-x_{4} & x_{3} & -x_{2} & x_{1} & x_{8} & -x_{7} & x_{6} & -x_{5} \\
-x_{5} & -x_{6} & -x_{7} & -x_{8} & x_{1} & x_{2} & x_{3} & x_{4} \\
-x_{6} & x_{5} & -x_{8} & x_{7} & -x_{2} & x_{1} & -x_{4} & x_{3} \\
-x_{7} & x_{8} & x_{5} & -x_{6} & -x_{3} & x_{4} & x_{1} & -x_{2} \\
-x_{8} & -x_{7} & x_{6} & x_{5} & -x_{4} & -x_{3} & x_{2} & x_{1}
\end{array}\right)
\end{aligned}
$$

Note that $\mathcal{G}_{2}$ is complex whereas $\mathcal{G}_{4}$ and $\mathcal{G}_{8}$ are real. The determinants of $\mathcal{G}_{2}, \mathcal{G}_{4}$, and $\mathcal{G}_{8}$ can be determined to be $\operatorname{det}\left(\mathcal{G}_{2}\right)=\left|x_{1}\right|^{2}+\left|x_{2}\right|^{2}, \operatorname{det}\left(\mathcal{G}_{4}\right)=\left(\sum_{i=1}^{4} x_{i}^{2}\right)^{2}$, and $\operatorname{det}\left(\mathcal{G}_{8}\right)=\left(\sum_{i=1}^{8} x_{i}^{2}\right)^{4}$. Obviously, all three codes achieve full diversity.

\section{B. New full-rate full-diversity codes from cyclic design}

Based on the determinant criterion, we can design the following linear real cyclic code

$$
\mathcal{H}_{4}=\left(\begin{array}{cccc}
x_{1} & x_{2} & x_{3} & x_{4} \\
x_{4} & x_{1} & x_{2} & -x_{3} \\
-x_{3} & -x_{4} & x_{1} & x_{2} \\
-x_{2} & x_{3} & x_{4} & x_{1}
\end{array}\right)
$$

whose determinant is obtained to be $\operatorname{det}\left(\mathcal{H}_{4}\right)=\left(x_{1}^{2}+x_{3}^{2}\right)^{2}+$ $\left(x_{2}^{2}+x_{4}^{2}\right)^{2}$. Thus, this code achieves full diversity for real symbols. From (2), it is easy to recognize that the non-zero offdiagonal elements in $\mathcal{G}^{H} \mathcal{G}$ represent the ISI terms. It should be mentioned that for the nonlinear codes proposed in [6], the diagonal elements, as will be shown later in Section IV, may cause ISI. Eq. (2) also explains why existing linear orthogonal codes are ISI free. Although the two codes $\mathcal{H}_{4}$ and $\mathcal{G}_{4}$ have the same diversity order, ISI degrades the performance of $\mathcal{H}_{4}$.

To design complex cyclic codes, we minimize ISI based on the structure of $\mathcal{H}_{4}$. Equivalently, we must maximize the number of columns that are pairwise orthogonal in the code matrix. Based on this design goal, a complex cyclic code for 4 transmit antennas is obtained as

$$
\mathcal{T}_{4}=\left(\begin{array}{cccc}
x_{1} & x_{2} & x_{3}^{*} & x_{4}^{*} \\
x_{4} & x_{1} & x_{2}^{*} & -x_{3}^{*} \\
-x_{3} & -x_{4} & x_{1}^{*} & x_{2}^{*} \\
-x_{2} & x_{3} & x_{4}^{*} & x_{1}^{*}
\end{array}\right) .
$$

In matrix $\mathcal{T}_{4}$, the first column is orthogonal to the third column, and the second column is orthogonal to the fourth column. It can be verified by using the rank criterion that this code provides full diversity for QPSK signals.

Let us compare the code from cyclic design given in (3) with the quasi-orthogonal code given in [3]

$$
\mathcal{A}_{4}=\left(\begin{array}{cccc}
x_{1} & x_{2} & x_{3} & x_{4} \\
-x_{2}^{*} & x_{1}^{*} & -x_{4}^{*} & x_{3}^{*} \\
-x_{3}^{*} & -x_{4}^{*} & x_{1}^{*} & x_{2}^{*} \\
x_{4} & -x_{3} & -x_{2} & x_{1}
\end{array}\right) .
$$

It follows easily that

$$
\mathcal{T}_{4}^{H} \mathcal{T}_{4}=\left(\begin{array}{cccc}
a & b_{1} & 0 & b_{2} \\
b_{1}^{*} & a & -b_{2} & 0 \\
0 & -b_{2}^{*} & a & b_{1}^{*} \\
b_{2}^{*} & 0 & b_{1} & a
\end{array}\right)
$$

and

$$
\mathcal{A}_{4}^{H} \mathcal{A}_{4}=\left(\begin{array}{cccc}
a & 0 & 0 & b_{3} \\
0 & a & -b_{3} & 0 \\
0 & -b_{3}^{*} & a & 0 \\
b_{3}^{*} & 0 & 0 & a
\end{array}\right)
$$

$a=\left|x_{1}\right|^{2}+\left|x_{2}\right|^{2}+\left|x_{3}\right|^{2}+\left|x_{4}\right|^{2}, \quad b_{1}=x_{1}^{*} x_{2}+x_{1} x_{4}^{*}+$ $x_{3}^{*} x_{4}-x_{2}^{*} x_{3}, \quad b_{2}=x_{1}^{*} x_{4}^{*}-x_{3}^{*} x_{4}^{*}-x_{2}^{*} x_{3}^{*}-x_{1}^{*} x_{2}^{*}, \quad b_{3}=$ $x_{1}^{*} x_{4}+x_{1} x_{4}^{*}-x_{2} x_{3}^{*}-x_{2}^{*} x_{3}$. Terms $b_{1}$ and $b_{2}$ are the ISI terms for the proposed code, and $b_{3}$ is the ISI term for the quasiorthogonal code. For QPSK symbols, $b_{1}$ and $b_{2}$ do not cause a loss in the diversity of the proposed cyclic code, but $b_{3}$ reduces the diversity order of the quasi-orthogonal code. Note that $b_{3}$ can be expressed as $b_{3}=f\left(x_{1}, x_{4}\right)+f\left(x_{2}, x_{3}\right)$, a sum of two independent functions. Therefore, we can decode $\left(x_{1}, x_{4}\right)$ and $\left(x_{2}, x_{3}\right)$ independently for the quasi-orthogonal code without degrading performance. For the cyclic code, however, the decoding complexity cannot be reduced even though there is no ISI between $x_{1}$ and $x_{3}$ or between $x_{2}$ and $x_{4}$.

\section{Numerical Results AND Discussion}

In this section, we simulate the performance of the proposed cyclic code, and compare it with that of the quasi-orthogonal code and the nonlinear code given in [6]. The nonlinear 


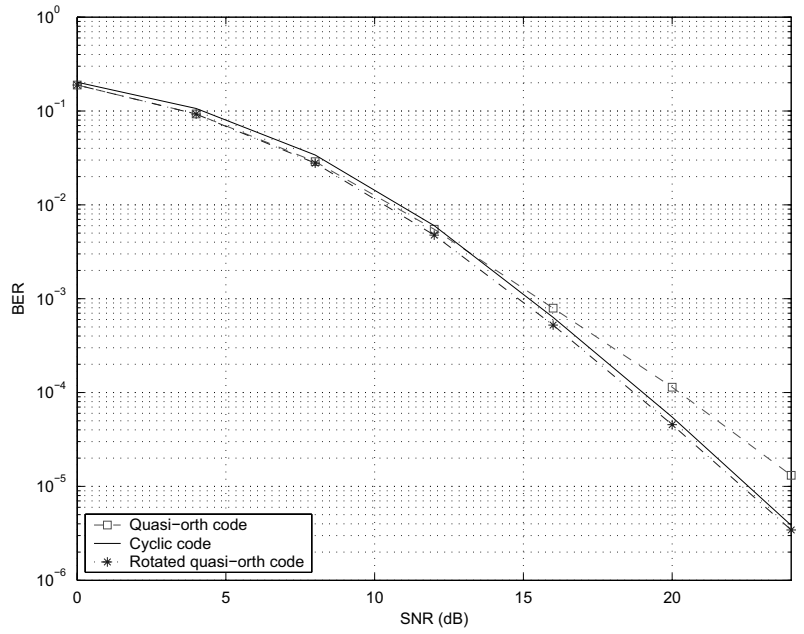

Fig. 1. Error performance of various schemes ( $N=3, M=1$, QPSK).

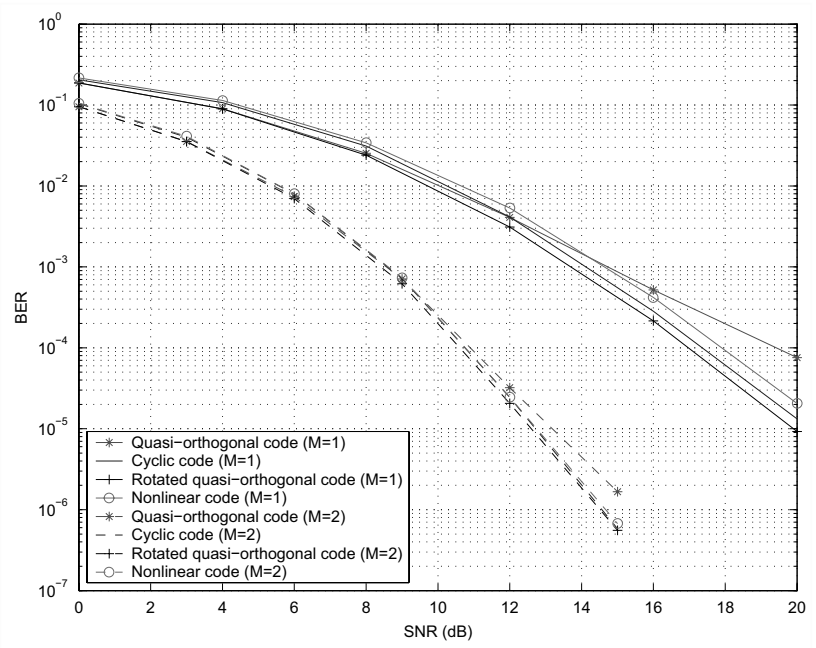

Fig. 2. Error performance of various schemes $(N=4, M=1,2$, QPSK).

orthogonal code given in [6] also provides full rate and full diversity for QPSK signals. Its code matrix is given as

$$
\mathcal{O}_{4}=\left(\begin{array}{cccc}
x_{1} & x_{2} & x_{3} & x_{4} \\
-x_{2}^{*} & x_{1}^{*} & -x_{4}^{*} & x_{3}^{*} \\
-z_{1}^{*} & z_{2} & x_{1}^{*} & -x_{2} \\
-z_{2}^{*} & -z_{1} & x_{2}^{*} & x_{1}
\end{array}\right)
$$

where $z_{1}=\operatorname{Re}\left\{x_{3}\right\}+j \operatorname{Im}\left\{2 x_{1} x_{2} x_{4}^{*}\right\}$ and $z_{2}=x_{1}^{* 2} x_{4}+$ $x_{2}^{2} x_{4}^{*}+x_{1}^{*} x_{2} x_{3}-x_{1}^{*} x_{2} x_{3}^{*}$. The orthogonality of $\mathcal{O}_{4}$ is achieved by introducing nonlinearity. As a result, the diagonal elements of $\mathcal{O}_{4}^{H} \mathcal{O}_{4}$ cause ISI. The nonlinear code requires the constellation to be $(1 / \sqrt{2}) e^{j\left(\frac{\pi}{4}+k \frac{\pi}{2}\right)}, k=0, \cdots, 3$.

Fig. 1 shows the error performance curves of the proposed code, the quasi-orthogonal code, and the quasi-orthogonal code with optimal constellation rotation (optimal angle is $\pi / 6$, see [4]) for a QPSK system with three transmit antennas and one receive antenna operating at $2 \mathrm{~b} / \mathrm{s} / \mathrm{Hz}$. Code matrices for the quasi-orthogonal design and for the cyclic design are chosen as, respectively, the first three columns of $\mathcal{A}_{4}$ and the first three columns of $\mathcal{T}_{4}$. Error performance results of various codes including the nonlinear code proposed in [6] for a QPSK system with four transmit antennas, one and two receive antennas operating at $2 \mathrm{~b} / \mathrm{s} / \mathrm{Hz}$ are shown in Fig. 2 .

It is observed from Figs. 1 and 2 that the slopes of SNR versus bit-error-rate curves of the proposed cyclic code and the quasi-orthogonal code with optimal constellation rotation are the same. Therefore, both codes achieve the same diversity order. The quasi-orthogonal code without rotation is found to provide only partial diversity. The cyclic code always provides better performance than the nonlinear code. In the low SNR region, quasi-orthogonal codes (with or without constellation rotation) perform slightly better than the cyclic code because quasi-orthogonal codes have less ISI (see (4) and (5)). At high SNR values, the cyclic code outperforms the unrotated quasiorthogonal code. The quasi-orthogonal code with optimal constellation rotation performs slightly better than the cyclic code. It is also observed that the three types of codes with full diversity have comparable performance when there are two receive antennas.

\section{CONClusion}

We proposed cyclic space-time block codes that achieve full rate and full diversity for QPSK systems with three or four transmit antennas. These codes perform much better than unrotated quasi-orthogonal codes at high signal-to-noise ratios, and perform slightly better than the nonlinear orthogonal code. Compared with the nonlinear code given in [6], the proposed cyclic code has a lower encoding complexity (decoding complexity is the same) and always performs better. The proposed code construction method could be applied to design rateone, full-diversity, complex cyclic codes for systems with an arbitrary number of transmit antennas.

\section{REFERENCES}

[1] V. Tarokh, H. Jafarkhani, and A. R. Calderbank, "Space-time block codes from orthogonal designs," IEEE Trans. Inform. Theory, vol. 45, pp. 14561467, July 1999.

[2] S. M. Alamouti, "A simple transmit diversity technique for wireless communications," IEEE J. Select. Areas Commun., vol. 16, pp. 14511458, Oct. 1998.

[3] H. Jafarkhani, "A quasi-orthogonal space-time block code," IEEE Trans. Commun., vol. 49, pp. 1-4, Jan. 2001.

[4] N. Sharma and C. B. Papadias, "Improved quasi-orthogonal codes through constellation rotation," IEEE Trans. Commun., vol. 51, no. 3, pp. 332-335, Mar. 2003.

[5] N. Sharma and C. B. Papadias, "Full rate full diversity linear quasiorthogonal space-time codes for any transmit antennas," in Proc. of Allerton Conference on Communications, Control and Computing, Oct. 2003, pp. 1193-1202.

[6] L. He and H. Ge, "A new full-rate full-diversity orthogonal space-time block coding scheme," IEEE Commun. Lett., vol. 7, pp. 590-592, Dec. 2003.

[7] V. Tarokh, N. Seshadri, and A. R. Calderbank, "Space-time codes for high data rate wireless communication: performance criterion and code construction," IEEE Trans. Inform. Theory, vol. 44, pp. 744-765, Mar. 1998. 